\title{
Acquired heterosubtypic antibodies in human immunity for avian H5N1 influenza
}

\author{
Garry W Lynch ${ }^{1,2,3,}$, Paul Selleck ${ }^{4}$ and John S Sullivan ${ }^{1,5}$ \\ ${ }^{1}$ Biosafety, Immunobiology, Global Health and Pandemic Infections Research, Central Clinical School, Faculties of \\ Medicine and ${ }^{2}$ Veterinary Science, The University of Sydney, Camperdown, NSW 2006, Australia, ${ }^{3}$ Cellular and \\ Molecular Pathology Research Unit, Oral Pathology and Oral Medicine, Westmead Centre for Oral Health, Westmead \\ Hospital and Faculty of Dentistry, University of Sydney, Westmead, NSW 2145, Australia, ${ }^{4}$ The Australian Animal \\ Health Laboratories, CSIRO, Geelong, Vic 3219, Australia, ${ }^{5}$ Children's Cancer Institute Australia for Medical Research, \\ Randwick, NSW 2031, Australia
}

*Correspondence to: Garry Lynch, Email: garry_lynch@optusnet.com.au, Tel: +61 29519 8151, Fax: +61 295198151

Received 31 August 2009, Revised 07 December 2009, Accepted 09 December 2009, Published online 15 December 2009

J Mol Genet Med (2009), 3(2), 205-209

(C) Copyright The Authors: This is an open access article, published under the terms of the Creative Commons Attribution Non-Commercial License (http://creativecommons.org/licenses/by-nc/2.0/uk/). This license permits noncommercial use, distribution and reproduction of the article, provided the original work is appropriately acknowledged with correct citation details.

\begin{abstract}
Well understood are the adaptive and dramatic neutralizing homosubtypic antibody responses to hypervariable, immunodominant sites of the hemagglutinin (HA) and neuraminidase (NA) of individual influenza strains. These define influenza subtypes and vaccines modelled upon their HA and NA antigens provide seasonal neutralizing antibody protection against subsequent exposure to the strain and its close relatives, but give little if any protection against antigenically drifted or shifted strains. Contrasting to this is a different form of acquired antibody response, called heterosubtypic immunity. This provides a more seasoned adaptive antibody response to immunerecessive epitopes that are highly-conserved amongst strains. Although, such responses are of lower individual amplitudes than seasonal mechanisms they are active across influenza subtypes, and may give pre-emptive protection against new strains yet to emerge. Heterosubtypic immunities have been well studied in animals, but surprisingly there is minimal evidence for this type of antibody immunity in humans. Thus championed is the notion that seasoned humoral responses can through repeated exposure to sites widely conserved across different strains, cumulatively provide humans with a level of broad protection against emergent novel strains, such as $\mathrm{H} 5 \mathrm{~N} 1$, that is not afforded by seasonal humoral responses.
\end{abstract}

KEYWORDS: Influenza, H5N1, H1N1, pandemic, immunity, heterosubtypic, antibody

\section{INTRODUCTION}

With a mortality up to 250 times greater than strains that cause epidemics the lethality of the 1918 avian H1N1 influenza pandemic is frequently reported (Palese et al, 2006; Taubenberger and Morens, 2006), but seldom appreciated is the fact that the majority $(>97 \%)$ of those infected survived, amounting to $0.8-2$ billion lives recovered. The most profound defence against influenza is provided by adaptive immunity that is mediated by neutralizing strain-specific "seasonal" antibodies directed against highly variable naïve antigens of an infecting influenza. But this is a post-emergent process that optimally takes weeks to months for full development (Murphy et al, 1982; Couch and Kasel, 1983; Edwards et al, 1986), and consequently beyond the time-frame of primary infection, viral shedding and disease development and is incapable of protecting against the first appearance 
of a new influenza strain. Therefore, additional mechanisms of protection need to be invoked to explain the mass herd immune protection of the community. Clearly, innate and heterosubtypic cellular immune mechanisms are involved in this; however, these alone are insufficient to justify this level of protection against a pandemic influenza, and prompt the issue of whether acquired heterotypic antibody immunity may provide a missing link in immune protection. Heterosubtypic cellular and antibody immune responses have been realized and studied for almost fifty years (Kilbuorne and Schulman, 1965), with extensive examination in animal models of influenza infection (reviewed in, Epstein, 2003; Grebe et al, 2008). There has been considerable study and demonstration of heterosubtypic cell-mediated immunity in humans as an essential component of systematic immune protection, and recent evaluation of its crossprotective vaccine potential (Doherty and Kelso, 2008). However, this is not the focus of this present review. In striking contrast to cell-mediated responses, until recently, there has been minimal study or direct evidence for heterosubtypic antibody immunity in humans, or resolution of protective heterosubtypic antibodies, or their partnering conserved influenza epitopes (i.e., protectopes; Plotnicky-Gilquin et al, 1999) (Bui et al, 2007; Ekiert et al, 2009; Sui et al, 2009). It is, therefore, proposed that akin to the heterosubtypic processes shown in animals, human antibody memories become acquired with age, from repeated infections and stimulus by highly-conserved influenza antigens. Despite being immunorecessive, this systematically leads to a broad "seasoned" heterosubtypic protection from antibodies that target equivalent sites of new strains. Thus it is important to identify whether or not such core antibody responses across influenzas do in fact provide significant immune protection against novel influenzas, such as avian H5N1 and evolving 2009 H1N1.

H5N1 influenza has had a higher measured mortality than the $1918 \mathrm{H} 1 \mathrm{~N} 1$ influenza, albeit with recovery of approximately $40 \%$ of individuals surviving infection. Epidemiologic studies indicate an age-related protection with survival of approximately $60 \%$ of individuals over the age of 40 years compared to less than $25 \%$ for individuals in the 10-20 years age group (Smallman-Raynor and Cliff, 2007). Similarly data from the $1918 \mathrm{H} 1 \mathrm{~N} 1$ and $1957 \mathrm{H} 2 \mathrm{~N} 2$ pandemics also indicate a greater level of protection in adults (Luk et al, 2001; Epstein, 2006), which may have reduced the impact in the adult sub-population older than 65 years to that of seasonal epidemics. While apparent protection of the elderly may result from earlier immunization of this cohort from exposure to a like virus, we believe it is equally plausible to result from a lifetime acquired heterosubtypic immunity, or even a combination of both. As seasonal antibodies provide the main correlate of homotypic neutralizing protection against influenza (Schild et al, 1975; Virelizier, 1975; Delem and Jovanovic, 1978; Kashyap et al, 2008), we now raise the question of whether seasoned, cross-reactive antibodies similarly provide a significant contribution for heterosubtypic protection. Certainly, our own findings implicate a preexisting pool of anti-influenza antibodies in an unexposed Australian population that can protect against avian $\mathrm{H} 5 \mathrm{~N} 1$ influenza, in vitro, along with H5-hemagglutinin binding antibodies that are most prominent with adult age (Lynch et al, 2008; Stelzer-Braid et al, 2008). This is also supported by observations of cross-reactive anti-neuraminidase antibodies from unexposed humans that partially protect H5N1 infected mice (Sandbulte et al, 2007). The idea of a role for low levels of pre-existing crosstypic antibodies in some humans to protect against foreign emerging influenza strains is indeed attractive. This would be supported by the other arm of heterosubtypic immunity, namely human cross-typic cell-mediated immunity, for which there is far more substantial evidence of protection against $\mathrm{H} 5 \mathrm{~N} 1$ pathogenesis and death (Jameson et al, 1999; Kreijtz et al, 2008; Lee et al, 2008; Roti et al 2008). A general overview of the combined human cellular and humoral system arms of heterosubtypic immunity and support evidence is provided in Figure 1.

The indication that heterosubtypic H5N1 neutralizing antibodies in unexposed individuals could be bolstered by a seasonal vaccine (Gioia et al, 2008) is encouraging. However, in general seasonal vaccines have not been reported to give wider immunity for emerging strains, such as the avian H5N1, or the $2009 \mathrm{H} 1 \mathrm{~N} 1$ pandemic influenzas (Kelly and Grant, 2009). We believe that this marked discrepancy is mainly due to the nature and size of the protective responses. Seasonal vaccines promote a substantial neutralizing response, mainly directed against the globular head region of hemagglutinin (HA) for cell receptor binding and neuraminidase (NA) for viral egress from an infected cell. In contrast, seasoned responses would by definition promote many lesser responses to multiple protein sites, including most, if not all, surface and interior proteins of influenza viruses. We predict this would individually go undetected, or be in the 'grey zone' of current in vitro assays. However, we propose that the net effect of marshalling the combined heterosubtypic systems responses would result in in vivo protection that could be measured and modelled. As a result we suggest that although seasonal and seasoned responses may appear discordant they would effectively champion one another, and ultimately enable both specific and universal protective capabilities to ideally reduce the debilitating and lethal effects of influenza to at least one of mild illness.

The requirements for vaccines to promote heterosubtypic antibody responses to conserved sites of influenza proteins for broad, seasoned, possibly universal protection would need to be inherently different from current seasonal vaccines: The latter express hypervariable determinants and promote high-tire antibody responses to homotypic immune dominant determinants of the HA globular-head to block cell receptor binding and neutralize infection. The challenge therefore is to separately design seasoned vaccines capable of promoting broad heterosubtypic protection against drifted and new zoonotic (avian) strains. Unlike seasonal vaccines that disrupt influenza-cell binding, a combination of seasoned vaccine approaches would likely be required to stimulate both antibody and Tcell responses to multiple conserved epitopes widely expressed across human and avian (e.g., H5N1) influenzas, and be accessible to antibodies or drugs to disrupt all aspects of the infection. Valuable insights 


\section{Heterosubtypic Human Immunity to Avian (H5N1) Influenza}

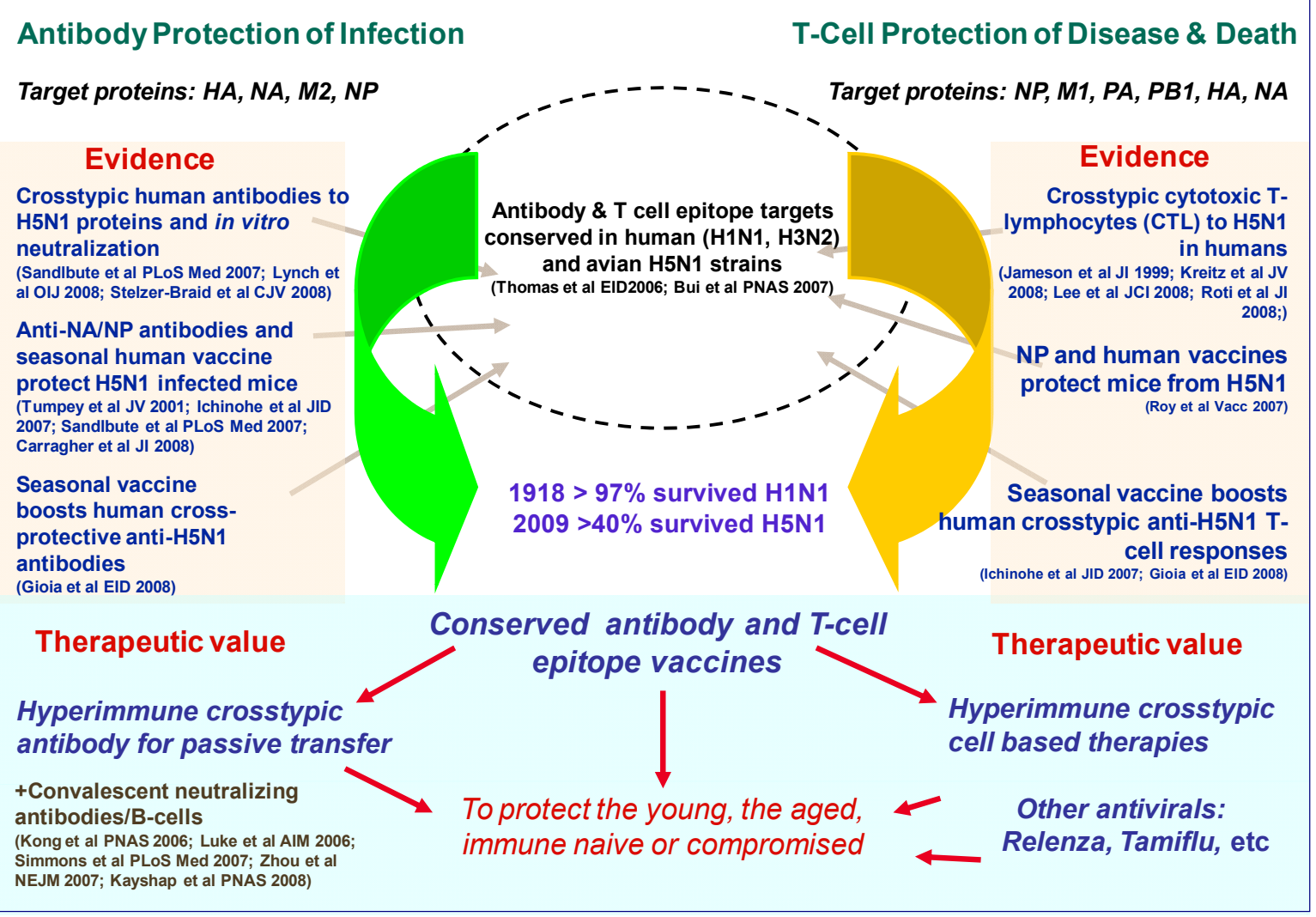

Figure 1. Schematic representation of the humoral and cell-mediated systems of heterosubtypic immunity for the delivery of crossstrain protection of novel influenzas, such as avian $\mathrm{H} 5 \mathrm{~N} 1$, via the targeting of cell-free virions and influenza protein expressing infected-cells. Both arms work in concert to protect against viral infection and propagation, pathogenesis and death. Shown is an overview of the respective mechanisms, the proteins they target, evidence of their cross-protective power, and avenues for the development of heterosubtypic-based vaccine and antiviral interventions for broad based influenza protection. [References: Jameson et al, 1999; Tumpey et al, 2001; Kong et al, 2006; Luke et al, 2006; Ichinohe et al, 2007; Roy et al, 2007; Sandlbute et al, 2007; Simmons et al, 2007; Zhou et al, 2007; Carragher et al, 2008; Gioia et al, 2008; Kayshap et al, 2008; Kreitz et al, 2008; Lee et al, 2008; Lynch et al, 2008; Roti et al, 2008; Stelzer-Braid et al, 2008]

into conserved linear and conformational antibody and $\mathrm{T}$ cell epitopes of human and avian influenza proteins (e.g., HA, NA, M2) are tabulated in the bioinformatic studies of $7 \mathrm{H} 1 \mathrm{~N} 1,8 \mathrm{H} 3 \mathrm{~N} 2$ and $2 \mathrm{H} 5 \mathrm{~N} 1$ isolates (Bui et al, 2007). In this analysis remarkably all but one Ab-epitope study was from animal experiments, but since then additional human antibody protectopes are being realized (Ekiert et al, 2009; Sui et al, 2009). This study was followed by a more encompassing evaluation of highly-conserved linear sites of 9 amino acids or longer, across all the strains of influenza (Heiny et al, 2007). Both studies revealed considerable conservation of sequence and antigen epitopes across the breadth of influenza proteins, with some 90 to $100 \%$ conserved and some already identified from animal studies as protective determinants (Bui et al, 2007). For seasoned vaccine initiatives the mapping and in vivo evaluation of epitope combinations for multiple-point targeting of infection is now necessary for vaccine design.

Examples of protective antibodies against conserved epitopes include those binding to the M2 protein tetrameric ectodomain (De Filette et al, 2008), site(s) on NA that disrupt sialidase release of budding virons from infected cells (Sandbulte et al, 2007) and of the HA stem that contains the proteolytic cleavage site and hydrophobic membrane fusion segment (Bui et al, 2007; Sui et al, 2009; Ekiert et al, 2009). In natural infections and vaccine antibody responses the conserved but poorly-immunogenic sites of the HA stalk must compete with the highlyimmunogenic and variable epitopes of the globular-head. Recent studies have shown the broadly protective value of human antibodies that target the pre-fusion structures of the HA stalk (Ekiert et al, 2009; Sui et al, 2009). As crossprotective antibodies to the conserved stalk are developed in mice following immunization with constructs with deleted globular-head but not with complete HA, studies are now needed to evaluate heterotypic humoral responses to a variety of conserved influenza epitopes in the absence of competing HA immune-dominant globular-head antigens (Sagawa et al, 1996). More contentious is what role if any do antibodies directed at internal influenza antigens, such as in the highly-conserved NP protein, play in hetero(sub)typic protection. NP is expressed on the surface of influenza infected cells (Virelizier et al, 1977; Stitz et al, 1990; Prokudina and Semenova, 1991) and is a major target for cytotoxic T-lymphocytes (Stitz et al, 
1990; Prokudina and Semenova, 1991). However, early studies indicated that NP did not protect against influenza infection (Stitz et al, 1990). Substantial antibody responses are generated to internal proteins by natural infection or vaccination (Couch, 2003). In mice, following vaccination with recombinant NP, both non-neutralizing and protective anti-NP antibodies are generated that reduce influenza virus titres and reduce mortality in influenza-infected mice passively treated with NP hyperimmune serum (Carragher et al, 2008). Similarly in a chicken study model, passively transferred anti-NP antibodies (and to a lesser extent antibodies to the polymerase complex proteins PA1, PB1, PB2) were found to protect against infection with highlypathogenic H7N3 influenza (Shahzad et al, 2008). It is important to determine if such responses are translatable to humans, and what role anti-NP antibodies play in heterosubtypic protection elicited by NP vaccines (Epstein et al, 2005; Price et al, 2009). It is also of interest to evaluate whether antibodies to infected cells expressing internal viral antigens, such as NP, may add to and complement other cross-typic responses (e.g., to M2e and HA stalk/fusion domains).

Whether the rules of engagement and responses to the generation of protective heterosubtypic antibodies in humans mirror well-characterized immunodominant homotypic responses remains to be determined. Certainly a significant unknown is how to best present protectope antigens for vaccines to stimulate protective heterosubtypic antibody responses. This would be governed by a variety of factors, including the optimal selection of protein or peptide constructs, epitope combinations, presentation (e.g., with or without adjuvant) (Straight et al, 2008), route of administration (Price et al, 2009) and roles of antibody secreting plasma cell and memory B cell responses (Wrammert et al, 2008). The first point of order, however, needs to be a clear demonstration of the role and significance of heterosubtypic antibodies in cross-typic protection of humans for influenza. Certainly the exciting findings of Ekiert et al (2009) and Sui et al (2009) indicate the potential, but this needs to be further supported by a more comprehensive evaluation of the complement of conserved influenza epitopes.

Thus, for more universal protection new approaches are required to target the many sites that are conserved across influenza proteins. However, because the immune responses to those sites are subverted by immune-dominant HA, and to a lesser extent NA antigens that form the centre-point of seasonal vaccines, new style constructs are needed that actually avoid those immunodominant sites. It would therefore be informative to examine heterosubtypic antibody responses for multiple viral constructs devoid of the regions that are the main focus of seasonal vaccines. The challenge is to test and evaluate a broad sampling of conserved influenza epitope combinations, including already indicated antibody protectopes, to substantiate the value of seasoned heterosubtypic antibody responses in broad protection of drifted and shifted influenzas. Studies of linear conserved regions can be made using recombinant protein fragments, peptides or DNA constructs before graduating to conformationally maintained recombinant and native viral protein constructs for the more difficult study of discontinuous conserved epitopes. Cross-strain antibody immune response comparisons of seasonal inactivated and live attenuated vaccines versus seasoned conserved antigen constructs will reveal the differences and similarities in their capacities for heterosubtypic protection to drive future vaccine initiatives. We envisage that if true value can be unequivocally established, future seasoned protectope vaccines will be used for the population at large to separately complement not replace current seasonal strategies. Together, these vaccines will systematically provide antibody protection to reduce the impact of emerging pathogenic and pandemic influenzas and reduce the considerable health and economic burden of epidemic influenzas. With comprehensive and synergistic targeting of conserved antibody epitopes in conjunction with strategies already raised for stimulating heterosubtypic T-cell responses (Doherty and Kelso, 2008; Lee et al, 2008) we are confident that vaccines for sustained and synergistic crosstypic influenza protection will be realized. Continued evolution and expansion of avian to human $\mathrm{H} 5 \mathrm{~N} 1$ and human to human $2009 \mathrm{H} 1 \mathrm{~N} 1$ pandemic H1N1 virus, highlights the need for more universal vaccines to generate heterotypic protection for immune-naive individuals, and promote rapid heterosubtypic hyperimmune responses for passive therapies to meet ongoing and future challenges.

\section{ACKNOWLEDGMENTS}

This work was partially supported by a University of Sydney Bridging Support Grant.

\section{COMPETING INTERESTS}

None declared.

\section{REFERENCES}

Bui HH, Peters B, Assarsson E, Mbawuike I and Sette A. 2007. $\mathrm{Ab}$ and $\mathrm{T}$ cell epitopes of influenza $\mathrm{A}$ virus, knowledge and opportunities. Proc Natl Acad Sci USA, 104, 246-251.

Carragher DM, Kaminski DA, Moquin A, Hartson L and Randall TD. 2008. A novel role for non-neutralizing antibodies against nucleoprotein in facilitating resistance to influenza virus. J Immunol 181, 4168-4176.

Couch RB and Kasel JA. 1983. Immunity to influenza in man. Ann Rev Microbiol, 37, 529-549.

Couch RB. 2003. An overview of serum antibody responses to influenza virus antigens. Dev Biol (Basel), 115, 25-30.

De Filette M, Martens W, Roose K et al. 2008. An Influenza A Vaccine Based on Tetrameric Ectodomain of Matrix protein 2. J Biol Chem, 24, 6597-6601.

Delem A and Jovanovic D. 1978. Correlation between rate of infection and preexisting titer of serum antibody as determined by single radial hemolysis during and epidemic of influenza A/Victoria/3/75. J Infect Dis 137, 194-196.

Doherty PC and Kelso A. 2008. Toward a broadly protective influenza vaccine. J Clin Invest 118, 3273-3275.

Edwards KM, Snyder P, Thompson JM, Johnson PR and Wright PF. 1986. In vitro production of anti-influenza virus antibody after simultaneous administration of $\mathrm{H} 3 \mathrm{~N} 2$ and $\mathrm{H} 1 \mathrm{~N} 1$ coldadapted vaccines in seronegative children. Vaccine, 4, 50-54.

Ekiert DC, Bhabha G, Elsliger MA et al. 2009. Antibody Recognition of a Highly Conserved Influenza Virus Epitope. Science,324, 246-251.

Epstein SL, Kong WP, Misplon JA et al. 2005. Protection against multiple influenza A subtypes by vaccination with highly conserved nucleoprotein. Vaccine, 23, 5404-5410. 
Epstein SL. 2003. Control of influenza virus infection by immunity to conserved viral features. Expert Review Anti Infect Ther, 1, 627-638.

Epstein SL. 2006. Prior H1N1 influenza infection and susceptibility of Cleveland Family Study participants during the H2N2 pandemic of 1957: an experiment of nature. J Infect Dis, 193, 49-53.

Gioia C, Castilletti C, Tempestilli M et al. 2008. Cross-subtype immunity against avian influenza in persons recently vaccinated for influenza. Emerg Infect Dis, 14, 121-128.

Grebe KM, Yewdell JW and Bennink JR. 2008. Heterosubtypic immunity to influenza A virus: where do we stand? Microbes Infect, 10, 1024-1029.

Heiny AT, Miotto O, Srinivasan KN et al. 2007. Evolutionarily conserved protein sequences of influenza a viruses, avian and human, as vaccine targets. PLoS One, 2, e1190.

Ichinohe T, Tamura S, Kawaguchi A et al. 2007. Crossprotection against $\mathrm{H} 5 \mathrm{~N} 1$ influenza virus infection is afforded by intranasal inoculation with seasonal trivalent inactivated influenza vaccine. J Infect Dis, 196, 1313-1320.

Jameson J, Cruz J, Terajima M and Ennis FA. 1999. Human CD8+ and CD4+ T lymphocyte memory to influenza A viruses of swine and avian species. J Immunol 162, 7578-7583.

Kashyap AK, Steel J, Oner AF et al. 2008. Combinatorial antibody libraries from survivors of the Turkish H5N1 avian influenza outbreak reveal virus neutralization strategies. Proc Natl Acad Sci USA, 105, 5986-5991.

Kelly H and Grant K. 2009. Interim analysis of pandemic influenza (H1N1) 2009 in Australia: surveillance trends, age of infection and effectiveness of seasonal vaccination. Euro Surveill, 14, pii:19288.

Kilbourne ED and Schulman JL. 1965. The induction of broadened (multitypic) immunity with doubly antigenic influenza virus recombinants. Trans Assoc Am Physicians, 78, 323-333.

Kong WP, Hood C, Yang ZY et al. 2006. Protective immunity to lethal challenge of the 1918 pandemic influenza virus by vaccination. Proc Natl Acad Sci USA, 103, 15987-15991.

Kreijtz JH, de Mutsert G, van Baalen CA, Fouchier RA, Osterhaus AD, Rimmelzwaan GF. 2008. Cross-recognition of avian H5N1 influenza virus by human cytotoxic T-lymphocyte populations directed to human influenza A virus. J Virol, 82, 5161-5166.

Lee LY, Ha do LA, Simmons C et al. 2008. Memory T cells established by seasonal human influenza $\mathrm{A}$ infection cross-react with avian influenza A (H5N1) in healthy individuals. J Clin Invest, 118, 3478-3490.

Luk J, Gross P and Thompson WW. 2001. Observations on mortality during the 1918 influenza pandemic. Clin Infect Dis, $33,1375-1378$.

Luke TC, Kilbane EM, Jackson JL and Hoffman SL. 2006. Metaanalysis: convalescent blood products for Spanish influenza pneumonia: a future H5N1 treatment? Ann Intern Med, 145, 631-632.

Lynch GW, Selleck PW, Axell AM et al. 2008. Cross-Reactive anti-Avian H5N1 Influenza Neutralizing Antibodies in a Normal 'Exposure-Naïve' Australian Blood Donor Population. The Open Immunol J, 1, 13-19.

Murphy BR, Nelson DL, Wright PF et al. 1982. Secretory and systemic immunological response in children infected with live attenuated influenza A virus vaccines. Infect Immun, 36, 11021108.

Palese P, Tumpey TM and Garcia-Sastre A. 2006. What can we learn from reconstructing the extinct 1918 pandemic influenza virus? Immunity, 24, 121-124.

Plotnicky-Gilquin H, Goetsch L, Huss T et al. 1999. Identification of multiple protective epitopes (protectopes) in the central conserved domain of a prototype human respiratory syncytial virus G protein. J Virol, 73, 5637-5645.

Price GE, Soboleski MR, Lo CY et al. 2009. Vaccination focusing immunity on conserved antigens protects mice and ferrets against virulent $\mathrm{H} 1 \mathrm{~N} 1$ and $\mathrm{H} 5 \mathrm{~N} 1$ influenza A viruses. Vaccine, 27, 6512-6521.

Prokudina EN and Semenova NP. 1991. Localization of the influenza virus nucleoprotein: cell-associated and extracellular non-virion forms. J Gen Virol, 72 (Pt 7), 1699-1702.

Roti M, Yang J, Berger D, Huston L, James EA and Kwok WW. 2008. Healthy human subjects have CD4+ T cells directed against H5N1 influenza virus. J Immunol, 180, 1758-1768.

Roy S, Kobinger GP, Lin J et al. 2007. Partial protection against H5N1 influenza in mice with a single dose of a chimpanzee adenovirus vector expressing nucleoprotein. Vaccine, 25, 68456851

Sagawa H, Ohshima A, Kato I, Okuno Y and Isegawa Y. 1996. The immunological activity of a deletion mutant of influenza virus haemagglutinin lacking the globular region. J Gen Virol 77, 1483-1487.

Sandbulte MR, Jimenez GS, Boon AC, Smith LR, Treanor JJ and Webby RJ. 2007. Cross-Reactive Neuraminidase Antibodies Afford Partial Protection against H5N1 in Mice and Are Present in Unexposed Humans. PLoS Med, 4, e59 (2007).

Schild GC, Oxford JS and Virelizier JL. 1975. Immunity to influenza. Dev Biol Stand, 28, 253-272.

Shahzad MI, Naeem K, Mukhtar M and Khanum A. 2008. Passive immunization against highly pathogenic Avian Influenza Virus (AIV) strain H7N3 with antiserum generated from viral polypeptides protect poultry birds from lethal viral infection. Virol J, 5, 144.

Simmons CP, Bernasconi NL, Suguitan AL et al.2007. Prophylactic and therapeutic efficacy of human monoclonal antibodies against H5N1 influenza. PLoS Med, 4, 928-936.

Smallman-Raynor MC and Cliff AD. 2007. Avian Influenza A (H5N1) Age Disrtibution in Humans. Emerg Infect Dis, 13, 510-512.

Stelzer-Braid S, Wong B, Robertson $\mathrm{P}$ et al. 2008. A commercial ELISA detects high levels of human H5 antibody but cross-reacts with influenza A antibodies. J Clin Virol, 43, 241-243.

Stitz L, Schmitz C, Binder D, Zinkernagel R, Paoletti E and Becht H. 1990. Characterization and immunological properties of influenza A virus nucleoprotein (NP): cell-associated NP isolated from infected cells or viral NP expressed by vaccinia recombinant virus do not confer protection. J Gen Virol, 71 ( Pt 5), 1169-1179.

Straight TM, Ottolini MG, Prince GA and Eichelberger MC. 2008. Antibody contributes to heterosubtypic protection against influenza A-induced tachypnea in cotton rats. Virol J, 5, 44.

Sui J, Hwang WC, Perez S et al. 2009. Structural and functional bases for broad-spectrum neutralization of avian and human influenza A viruses. Nat Struct Mol Biol, 16, 265-273.

Taubenberger JK and Morens DM. 2006. 1918 Influenza: the mother of all pandemics. Emerg Infect Dis 12, 15-22.

Thomas PG, Keating R, Hulse-Post DJ and Doherty PC. 2006. Cell-mediated protection in influenza infection. Emerg Infect Dis, $12,48-54$.

Tumpey TM, Renshaw M, Clements JD and Katz JM. 2001. Mucosal delivery of inactivated influenza vaccine induces Bcell-dependent heterosubtypic cross-protection against lethal influenza A H5N1 virus infection. J Virol, 75, 5141-5150.

Virelizier JL, Allison AC, Oxford JS and Schild GC. 1977. Early presence of ribonucleoprotein antigen on surface of influenza virus-infected cells. Nature 266, 52-54.

Virelizier JL. 1975. Host defenses against influenza virus: the role of anti-hemagglutinin antibody. J Immunol 115, 434-439.

Wrammert J, Smith K, Miller J et al. 2008. Rapid cloning of high-affinity human monoclonal antibodies against influenza virus. Nature, 453, 667-671.

Zhou B, Zhong N and Guan Y. 2007. Treatment with convalescent plasma for influenza A (H5N1) infection. N Engl J Med, 357, 1450-1451. 